\title{
Translational and rotational velocities in shear-driven jamming of ellipsoidal particles
}

\author{
Yann-Edwin Keta ${ }^{1,2,3}$ and Peter Olsson $\circledast^{1}$ \\ ${ }^{1}$ Department of Physics, Umeå University, 90187 Umeå, Sweden \\ ${ }^{2}$ Département de Physique, École Normale Supérieure de Lyon, 69364 Lyon Cedex 07, France \\ ${ }^{3}$ Département de Physique, Université Claude Bernard Lyon 1, 69622 Villeurbanne Cedex, France
}

(Received 22 October 2019; revised 9 June 2020; accepted 5 November 2020; published 30 November 2020)

\begin{abstract}
We study shear-driven jamming of ellipsoidal particles at zero temperature with a focus on the microscopic dynamics. We find that a change from spherical particles to ellipsoids with aspect ratio $\alpha=1.02$ gives dramatic changes of the microscopic dynamics with much lower translational velocities and a new role for the rotations. Whereas the velocity difference at contacts - and thereby the dissipation-in collections of spheres is dominated by the translational velocities and reduced by the rotations, the same quantity is in collections of ellipsoids instead totally dominated by the rotational velocities. By also examining the effect of different aspect ratios we find that the examined quantities show either a peak or a change in slope at $\alpha \approx 1.2$, which thus gives evidence for a crossover between different regions of low and high aspect ratio.
\end{abstract}

DOI: 10.1103/PhysRevE.102.052905

\section{INTRODUCTION}

Dense collections of circular disks in two dimensions (2D) and spheres in 3D with contact interaction at zero temperature have been studied extensively during the past decades with the aim to understand the jamming transition. Since ordinary molecular dynamics that automatically explores phase space does not work at zero temperature one has mainly used two other computational methods. The first is what we call isotropic jamming, which is the generation of particle packings with energy-minimization methods in various ways [1-6] and the second is to model a shear flow by steadily shearing the system, either quasistatically [7-9] or at different constant shear rates [10-16].

Since real particles are seldom perfectly spherical an interesting generalization of this model with obvious experimental relevance is the change from spherical to aspherical particles. It has already been argued that the spherical limit is singular [17-20] and the purpose of the present paper is to explore further consequences of this change to aspherical particles.

In the present paper we do shearing simulations of both spherical and ellipsoidal particles to examine how the asphericity affects the microscopic dynamics. We do find dramatic effects. Focusing first on aspect ratio $\alpha=1.02$, we find that the ellipsoidal particles have considerably lower translational velocities and that their rotational motion gets a different role. Since the quartic modes found in static packings are primarily rotational in character $[21,22]$ we believe that these effects are consequences of the quartic modes on

Published by the American Physical Society under the terms of the Creative Commons Attribution 4.0 International license. Further distribution of this work must maintain attribution to the author(s) and the published article's title, journal citation, and DOI. Funded by Bibsam. the shear-driven dynamics. Our work thus focuses on quantities that have not been examined before in the context of shear-driven elongated particles, which until now have mostly been analyzed with focus on possible ordering of the particles $[17,20,23]$ and macroscopic quantities $[19,24,25]$. When doing simulations also with a set of larger aspect ratios we find several quantities to have features at $\alpha \approx 1.2$, which suggests the existence of different regions of high and low aspect ratio.

The organization of this paper is as follows. In Sec. II we start with an explanation of the origin of the quartic modes that have been found in packings of nonspherical particles; we then describe the model and the simulations behind the results in this paper. In Sec. III we consider almost spherical particles-particles with aspect ratio $\alpha=1.02$-where the key finding is that already such a small asphericity gives a very different behavior. In Sec. IV we examine the dependency on $\alpha$ and present evidence that several different quantities show a change in behavior at $\alpha \approx 1.2$. A summary and some concluding remarks are given in Sec. V.

Finally, in the Appendixes, we consider several related matters. In Appendix A we consider the effect of a certain modification of our model which has been argued to make it more physical; we find the effect to be very small. In Appendix B we give some more details behind the behavior of a key quantity of the paper. In Appendix $\mathrm{C}$ we extract a correlation coefficient from some data presented in one of the main sections to simplify comparisons with results in the literature. In Appendix D we present figures that illustrate the extrapolations to the low shear rate limit for some of the key quantities in the paper.

\section{MODEL AND SIMULATIONS}

\section{A. Ellipses and quartic modes}

A key result from the study of jamming of spherical particles is that jamming occurs when the average number of contacts per particle is just enough to stabilize the degrees of 


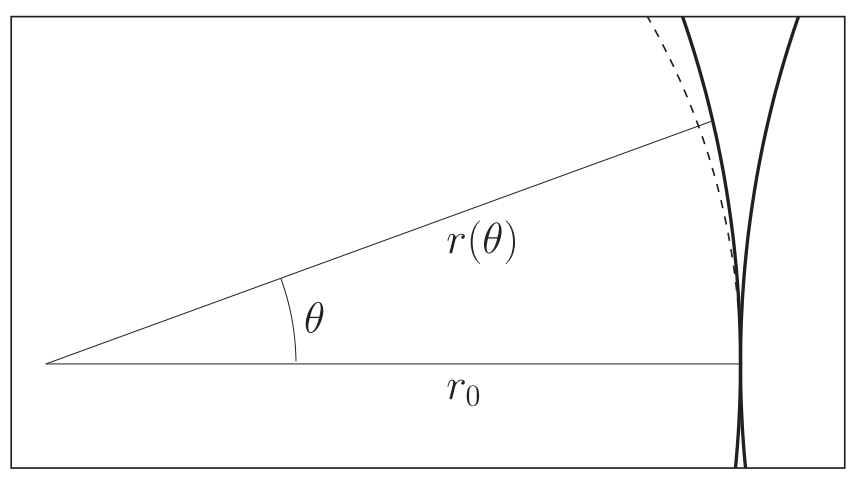

FIG. 1. Discussion of ellipses and the quartic modes. The thick solid lines are the ellipses, whereas the dashed line is a circle with radius $r_{0}$.

freedom, according to the isostatic condition, $z=2 d_{f}$ [26,27]. Since rotations become relevant for aspherical particles we find $d_{f}=6$ for general ellipsoids and $d_{f}=5$ for ellipsoids with a symmetry axis (spheroids), which is the kind of particles studied in the present work. If the isostatic conjecture were always valid one would expect a jump in $z$ already for a slight deviation from the spherical limit. It has however long been known that there is no jump in $z$ [28] and that the systems are thus hypostatic at jamming, i.e., have a smaller number of contacts than number of degrees of freedom.

To see how a hypostatic packing can be jammed it is helpful to consider a contact between two ellipses that barely touch at their respective waists. (We here consider ellipses for simplicity but the same reasoning should apply to ellipsoids.) This contact will then not only hinder the motion of the ellipses toward each other but will also hinder their respective rotations, thus affecting two different degrees of freedom for each particle. Whereas the interaction energy will be quadratic in the position coordinate it will instead be quartic-to the fourth power-in the angle coordinates. To show this, Fig. 1 shows a closeup of two ellipses, $x^{2}+a^{2} y^{2}=r_{0}^{2}$, with $a<1$, touching at their waists. The dashed curve is a part of a circle with radius $r_{0}$. To show that the relative distance between the circle and the ellipsoid depends quadratically on the angle $\theta, r(\theta) / r_{0}-1 \sim \theta^{2}$, we note that $x^{2}=r_{0}^{2}-a^{2} y^{2}$ in $r^{2}(\theta)=$ $x^{2}+y^{2}$ becomes

$$
r^{2}(\theta)=r_{0}^{2}+\left(1-a^{2}\right) y^{2} .
$$

For a small $\theta$ such that $y=r_{0} \theta$ this becomes

$$
r^{2}(\theta) / r_{0}^{2}=1+\left(1-a^{2}\right) \theta^{2},
$$

which finally gives

$$
r(\theta) / r_{0} \approx 1+\frac{1}{2}\left(1-a^{2}\right) \theta^{2} .
$$

The fourth power follows since the energy is in turn quadratic in the overlap. For a jammed collection of particles this mechanism leads to a set of quartic vibrational modes [21,22,29], beside the ordinary modes for which the energy is quadratic in the displacements. [For a particle with several contacts, not at their waists, $r(\theta)$ for each contact also has a term linear in the rotation $\theta$. In force-balanced jammed packings we expect these linear terms to cancel each other out, such that only the $\theta^{2}$-dependence remains.] From studies of static packings it has been found that the number of quartic modes exactly matches the deviation in contact number from the isostatic value [21].

\section{B. Model and simulations}

Our system is a bidisperse collection of particles with nominal diameters $d_{b}=1.4 d_{s}$, in equal proportion. The particles are prolate spheroids, i.e., ellipsoids with two equal minor axes with $d^{(1)}>d^{(2)}=d^{(3)}$. The aspect ratio is $\alpha=$ $d^{(1)} / d^{(2)}>1$. To make the particle volume independent of $\alpha$ we take the semiaxes to be given by $d^{(1)}=\alpha^{2 / 3} d$ and $d^{(2)}=$ $d^{(3)}=\alpha^{-1 / 3} d$, where $d=d_{b}, d_{s}$ for big and small particles. The method used to check for particle overlaps is described in Ref. [17]. For overlapping ellipsoids we define a scale factor $\delta<1$ such that the ellipsoids are just barely touching when they are rescaled by $\delta$, keeping the center of mass position fixed. The elastic force between overlapping particles is then given by $\mathbf{F}_{i j}^{\mathrm{el}}=k_{e} \delta(1-\delta) \hat{\mathbf{n}}_{i j} /\left[\left(\mathbf{r}_{i}-\mathbf{r}_{j}\right) \cdot \hat{\mathbf{n}}_{i j}\right]$, where $\hat{\mathbf{n}}_{i j}$ is a unit vector pointing inwards to particle $i$ at the point of contact with particle $j$.

We take a purely collisional dynamics where dissipation takes place at the particle contacts. With $\mathbf{r}_{i, j}^{C} \equiv \mathbf{r}_{j, i}^{C}$ the position of the point of contact of particle $i$ with particle $j-$ a point on the rescaled ellipsoids - the velocity of the surface of particle $i$ at this point is

$$
\mathbf{v}_{i}\left(\mathbf{r}_{i, j}^{C}\right)=\mathbf{v}_{i}+\mathbf{v}_{i}^{R}\left(\mathbf{r}_{i, j}^{C}\right)
$$

where $\mathbf{v}_{i}$ is the center of mass translational motion and $\mathbf{v}_{i}^{R}\left(\mathbf{r}_{i, j}^{C}\right)=\omega_{i} \times\left(\mathbf{r}_{i, j}^{C}-\mathbf{r}_{i}\right)$ is the velocity at the point of contact due to $\omega_{i}$, the rotational velocity of particle $i$. The velocity difference at that point is $\mathbf{v}_{i j}^{C}=\mathbf{v}_{i}\left(\mathbf{r}_{i, j}^{C}\right)-\mathbf{v}_{j}\left(\mathbf{r}_{i, j}^{C}\right)$ and the dissipative force is given by $\mathbf{F}_{i j}^{\mathrm{dis}}=-k_{d} \mathbf{v}_{i j}^{C}$. The equations of motion are

$$
\begin{gathered}
m_{i} \dot{\mathbf{v}}_{i}=\sum_{j}\left[\mathbf{F}_{i j}^{\mathrm{el}}+\mathbf{F}_{i j}^{\mathrm{dis}}\right], \\
\mathbf{I}_{i} \cdot \dot{\boldsymbol{\omega}}_{i}=\sum_{j}\left(\mathbf{r}_{i, j}^{C}-\mathbf{r}_{i}\right) \times\left[\mathbf{F}_{i j}^{\mathrm{el}}+\mathbf{F}_{i j}^{\mathrm{dis}}\right],
\end{gathered}
$$

where $\mathbf{I}_{i}$ is the moment of inertia tensor. We take $k_{e}=1$ and $k_{d}=1 / 2$ and simulate with $N=1024$ particles. The density (filling factor) is $\phi=N\left(d_{s}^{3}+d_{b}^{3}\right) \pi /\left(12 L^{3}\right)$. We take the unit of length to be equal to $d_{s}$, the unit of energy to be $k_{e}$, and the unit of mass to be $m_{s}$, and let the particle mass be proportional to the particle volume. The unit of time is $t_{0}=d_{s} \sqrt{m_{s} / k_{e}}$.

This model is one of the simplest with a reasonable dynamics but it is unusual in that it has no coupling between the tangential dissipative force and the strength of the elastic force. In dry friction the tangential dissipative force is related to the normal elastic force through the relation $F_{t}^{\text {dis }} \leqslant \mu F^{\mathrm{el}}$ and it is interesting to check if a finite $\mu$ changes the behavior. (Our model corresponds to an infinite $\mu$.) However, as shown in Appendix A, the introduction of a finite $\mu$ only gives minor changes, at the densities of interest, simply because the dissipating forces are anyway typically much smaller than the elastic ones. 

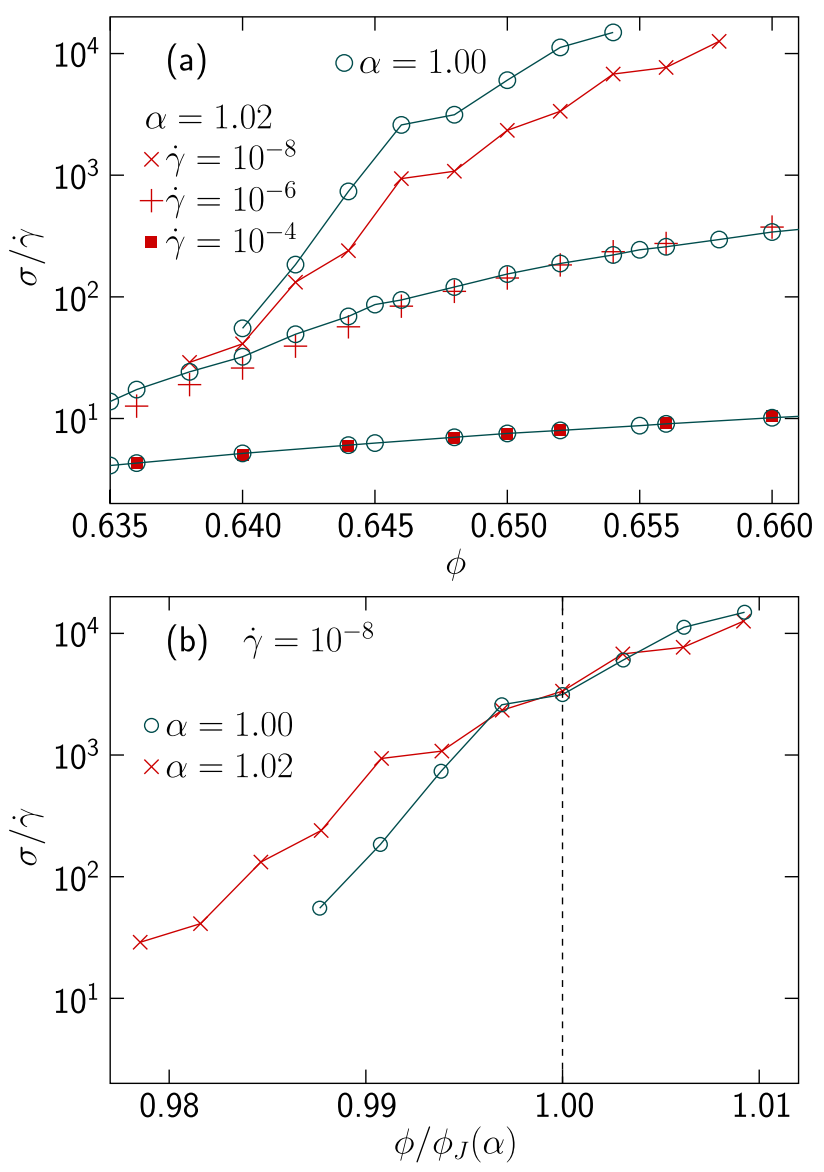

FIG. 2. Shear viscosity for spheres, $\alpha=1.00$, and ellipsoids, $\alpha=1.02$. Panel (a) shows direct comparisons for shear strain rates $\dot{\gamma}=10^{-8}, 10^{-6}$, and $10^{-4}$, which show that a small asphericity only has significant effects at low shear strain rates. Panel (b), which is the same data plotted vs $\phi / \phi_{J}(\alpha)$, where $\phi_{J}(1.00)=0.648$ and $\phi_{J}(1.02)=0.652$, shows that the change in $\sigma / \dot{\gamma}$ at small $\dot{\gamma}$ can be understood as an effect of a shift in jamming density. This has also been found to be the case for spherocylinders in 2D [19].

\section{RESULTS FOR A SMALL ASPHERICITY, $\alpha=1.02$}

\section{A. Crossover for slightly aspherical particles}

At high shear rates the particle overlaps are sizable and could well be comparable to the typical deviation of the particle surface from the spherical shape. When that is the case one expects aspherical particles to behave very similarly to the spherical ones. This is no longer the case at low shear strain rates where the overlaps are much smaller and the particles have time to rotate to pack more densely. That there are clear differences at low shear strain rates is also seen in the jamming density - which is a quantity that is characterized by the behavior in the limit of small shear strain rates - which has been found to increase linearly with the distance from the spherical point, $\phi_{J}(\alpha)-\phi_{J}(1) \sim|\alpha-1|$, in isotropic jamming [28]. One then expects the main effect of the asphericity close to jamming to be to shift the curves to (somewhat) higher $\phi$ [19].

This leads to the expectation of a crossover as the shear strain rate decreases, from a behavior as in spherical particles to a different behavior where the asphericity plays a role. This expection is confirmed in Fig. 2 which is shear viscosity for spheres and slightly aspherical particles, $\alpha=1.02$, for different shear strain rates, $\dot{\gamma}$. The figure shows that these two cases behave essentially the same at high $\dot{\gamma}$ but become quite different at lower $\dot{\gamma}$. The shear viscosity of the ellipsoids at lower $\dot{\gamma}$ is somewhat lower than for spheres, which is consistent with the jamming transition taking place at a higher density. Figure 2(b) shows that a rescaling of the density can accommodate this change, by displaying this data vs $\phi / \phi_{J}(\alpha)$. We do however stress that this is not the full story. A key message of the present paper is that there are big differences in the microscopic dynamics in spite of the small differences in certain macroscopic quantities.

\section{B. Translational and rotational velocities}

A study of the microscopic dynamics shows that the onset of particle asphericity also gives other effects than a shift of the jamming transition. A simple and direct way to investigate the dynamics is to determine the ratio of the nonaffine translational velocity and the rotational velocity, $v / \omega . v$ and $\omega$ are here the root-mean-square nonaffine translational velocity $\mathbf{v}_{i}^{\text {na }}=\mathbf{v}_{i}-\dot{\gamma} y_{i} \hat{x}$ and the root-mean-square rotational velocity $\boldsymbol{\omega}_{i}$, i.e., $v=\sqrt{\left\langle\left(\mathbf{v}_{i}^{\text {na }}\right)^{2}\right\rangle}$ and $\omega=\sqrt{\left\langle\omega_{i}^{2}\right\rangle}$. Figures 3(a) and 3(b) show the ratio $v / \omega$ vs $\phi$ for spherical and ellipsoidal particles at several different shear rates. For spherical particles, panel (a), this ratio is to a good approximation independent of $\dot{\gamma}$ and takes values from 0.4 through 0.9 in the density window of the figure. For ellipsoidal particles, panel (b), the behavior is similar to that of spheres at low densities but becomes very different at higher densities, with a dramatic shear rate dependence and a big drop. The ratio $v / \omega$ changes by a factor of about five for our range of shear rates. As this is for an asphericity $\equiv \alpha-1$ of only $2 \%$, the effect is surprisingly big. As shown in Appendix B the decrease in this ratio is largely an effect of a smaller $v$ but there is also a contribution from an increase in $\omega$.

To study the dependence on $\alpha$ in more detail we want to compare data at the respective jamming densities, $\phi_{J}(\alpha)$. For $\alpha=1.00$ we take $\phi_{J}(1.00) \approx 0.648$ [30] and for $\alpha=1.02 \mathrm{we}$ take $\phi_{J}(1.02)=0.652$. The estimate of $\phi_{J}(\alpha)$ is discussed in Sec. IV A. These are approximate values but it turns out that the (small) uncertainties in $\phi_{J}(\alpha)$ are not of any big concern since the studied quantities depend only slowly on $\phi$.

With the pronounced decrease of $v / \omega$ with decreasing $\dot{\gamma}$ in Fig. 3(b) it becomes interesting to try to extrapolate to the limit of vanishing shear rate. At $\phi_{J}(1.02)=0.652-$ which is also the density where $v / \omega$ has its minimum-we fit to constant plus algebraic behavior, $v\left(\phi_{J}, \dot{\gamma}\right) / \omega\left(\phi_{J}, \dot{\gamma}\right)=[v / \omega]_{0}+$ $a \dot{\gamma}^{b}$. As shown in Fig. 3(c) the fit is good and suggests that the limiting value is a finite constant, $[v / \omega]_{0} \approx 0.11$. This quantity is also shown for general $\alpha$ in Fig. 6(b).

\section{Rotations around different axes}

Slowly sheared spherical particles close to jamming display erratic translational displacements which are needed to fit into a constantly changing environment. The lower translational velocity of the ellipsoidal particles suggests a picture where this translational motion is largely replaced by rotations. Since only the rotations around the minor axes can help 

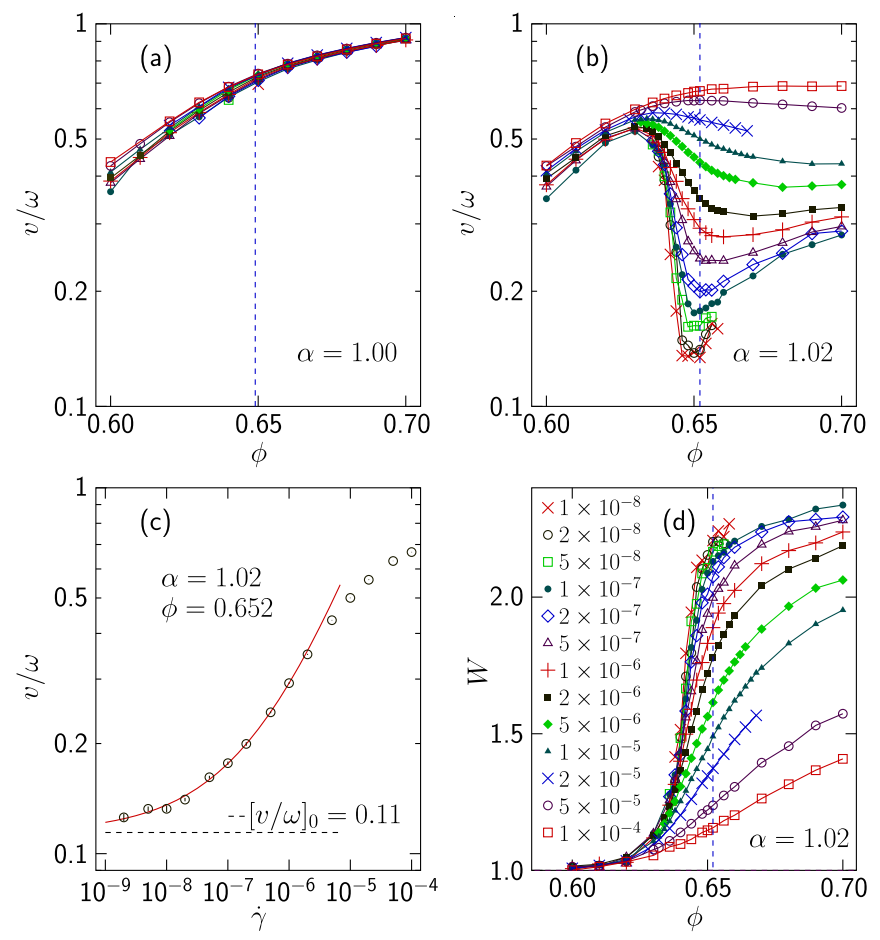

FIG. 3. Measures of translational and rotational particle velocities for spheres, $\alpha=1.00$, and ellipsoids with $\alpha=1.02$. Panels (a) and (b) show $v / \omega$ vs $\phi$ for spheres and ellipsoids. [Legends for the different $\dot{\gamma}$ are in panel (d).] For spheres this quantity only depends slowly on $\phi$ but for ellipsoids it decreases in a dramatic way, and even more so for the lower shear rates. The vertical dashed lines are the respective $\phi_{J}$. Panel (c) shows an extrapolation of $v / \omega$ at $\phi_{J}(1.02)$ to the $\dot{\gamma} \rightarrow 0$ limit, giving $[v / \omega]_{0} \approx 0.11$. Panel (d) shows $W$, which is the ratio of the root-mean-square rotational velocities along the minor axes and the major axis, as described in the main text. The figure shows that the rotations are predominantly around the minor axes, which are the ones that can help the particles fit in with their neighbors.

the particles fit in with their neighbors, one expects these rotations to be more prominent than the rotations around the major (symmetry) axis. With the rotation vector in the body frame given by $\widetilde{\boldsymbol{\omega}}=\left(\widetilde{\omega}^{(1)}, \widetilde{\omega}^{(2)}, \widetilde{\omega}^{(3)}\right)$, where the major axis is direction "1," Fig. 3(d) shows $W \equiv\left[\widetilde{\omega}_{\mathrm{rms}}^{(2)}+\widetilde{\omega}_{\mathrm{rms}}^{(3)}\right] / 2 \widetilde{\omega}_{\mathrm{rms}}^{(1)}$-from the respective root-mean-square values-vs $\phi$ for different $\dot{\gamma}$. At low densities this quantity is close to unity, indicating that the rotations are equally strong around the different semiaxes, but it increases to higher values around the jamming density. This increase is small for high $\dot{\gamma}$, reaches more than a factor of two for our lowest $\dot{\gamma}$, and an extrapolation at $\phi_{J}$, again by fitting to constant plus algebraic behavior, suggests the $\dot{\gamma} \rightarrow 0$ limit $W_{0}=2.25$.

\section{Velocity difference at particle contacts}

Whereas the quantities discussed above are single-particle quantities, we now turn to the velocity difference at particle contacts, and, more specifically, the role of rotations and translations for these velocity differences. Since the velocity difference is related to the dissipation, and thereby to the shear viscosity, this is a quantity with a more direct physical
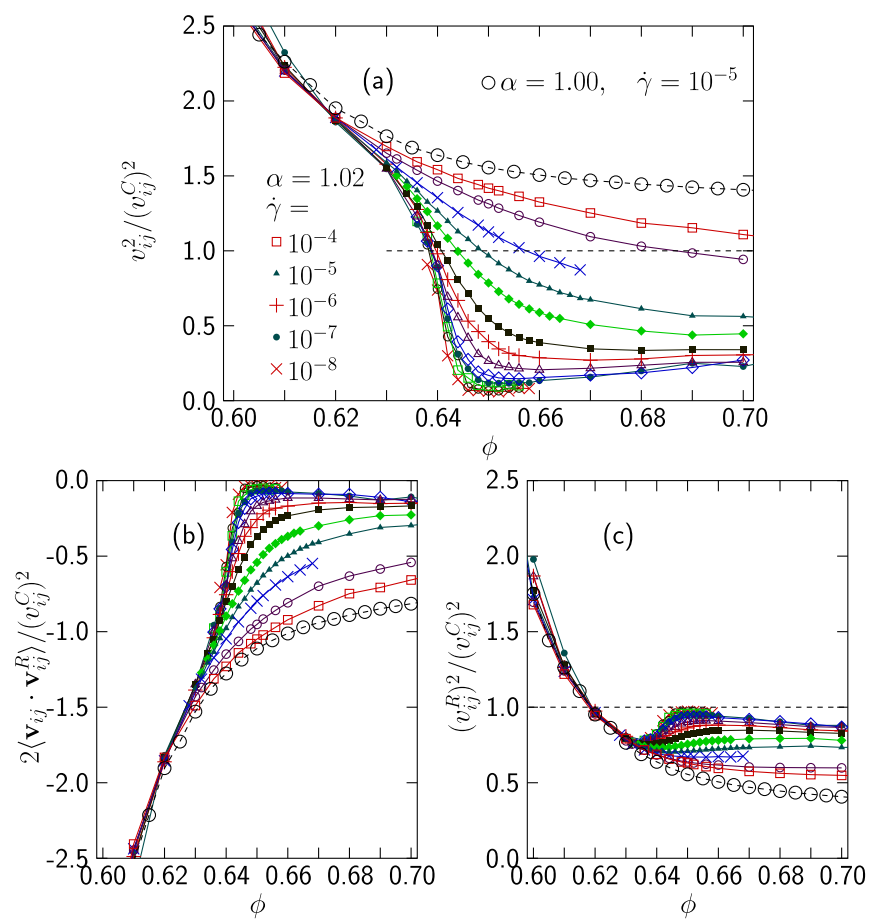

FIG. 4. Contributions of the different terms of Eq. (5) to $\left(v_{i j}^{C}\right)^{2}-$ and thus to the dissipation-for both spheres (open circles, $\dot{\gamma}=$ $10^{-5}$, no significant $\dot{\gamma}$ dependence) and ellipsoids for many different shear rates, $\dot{\gamma}=1 \times 10^{-8}, 2 \times 10^{-8}, 5 \times 10^{-8}, \ldots, 10^{-4}$. The figure shows the difference between spheres and ellipsoids in two ways. (i) For spheres the velocity difference is mainly due to the translational velocity difference and the effect of the rotations-panels (b) and (c) - is to reduce the velocity difference at contacts. (ii) For ellipsoids at densities around $\phi_{J}(1.02)=0.652$ and in the limit of small shear rates (crosses for $\dot{\gamma}=10^{-8}$ ) the velocity difference is mainly due to the rotational velocity. The translational velocity - panels (a) and (b) - gives a very small contribution.

relevance than the single particle velocities, and it is also the quantity that most clearly shows the difference between spherical and aspherical particles.

For the analysis we use the notation in and after Eq. (2) and write the contact velocity difference as the sum of translational and rotational parts, $\mathbf{v}_{i j}^{C}=\mathbf{v}_{i j}+\mathbf{v}_{i j}^{R}$. The contact velocity difference squared then becomes

$$
\left(v_{i j}^{C}\right)^{2}=v_{i j}^{2}+2\left\langle\mathbf{v}_{i j} \cdot \mathbf{v}_{i j}^{R}\right\rangle+\left(v_{i j}^{R}\right)^{2} .
$$

The relative contributions of these three terms are shown in Fig. 4 both for spheres (where the behavior is independent of $\dot{\gamma}$ ) and for ellipsoids at several different $\dot{\gamma}$. We then compare the behavior of ellipsoids for the lowest $\dot{\gamma}$ with the behavior of spheres. For spheres $v_{i j}^{C}$ is dominated by the translational velocities, $\mathbf{v}_{i j}$, and reduced by the rotations, $\mathbf{v}_{i j}^{R}$, which is seen by panels (b) and (c) together giving a negative contribution. The relative contributions of the three terms in Eq. (5) at $\phi=$ 0.648 are $1.57,-1.13$, and 0.56 .

For the ellipsoids the main contribution is from the rotations, $\mathbf{v}_{i j}^{R}$, and the contribution from $\mathbf{v}_{i j}$ is very small. This is seen by the crosses, which are data for $\alpha=1.02$ and $\dot{\gamma}=$ $10^{-8}$, in the three panels at $\phi_{J}$ being close to zero and unity, 

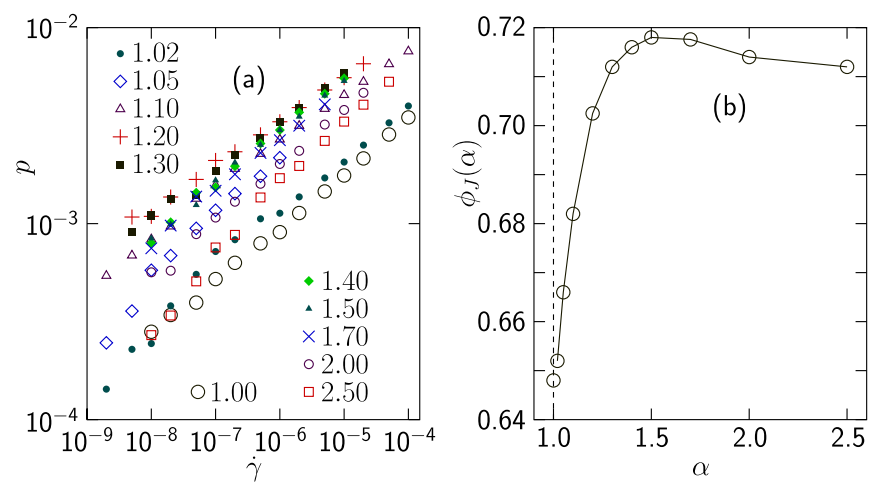

FIG. 5. Determination of our approximate $\phi_{J}(\alpha)$. Panel (a) shows $p(\dot{\gamma})$ for spheres (big open circles) and 10 different $\alpha>1$ at the respective $\phi_{J}(\alpha)$ chosen such that each data set is algebraic in $\dot{\gamma}$ to a decent approximation. Panel (b) is $\phi_{J}(\alpha)$.

respectively. After extrapolating the three terms of Eq. (5) at $\phi_{J}(1.02)=0.652$ to $\dot{\gamma} \rightarrow 0$ their relative contributions are found to be $0.04,-0.02$, and 0.98 , which shows that it is the rotations that totally dominate the velocity difference. The translations only contribute $4 \%$ of the dissipated power, whereas the rotations, when including the negative mixed term, contribute the remaining $96 \%$.

A different way to analyze the data of Fig. 4(b) is given in Appendix C. The focus is there on the correlation coefficient which is obtained with a different normalization of the same data, and it is found that the system changes from $\mathbf{v}_{i j}$ and $\mathbf{v}_{i j}^{R}$ being almost perfectly anticorrelated at low densities, $\phi<0.58$, to a very weak anticorrelation at $\phi_{J}$.

\section{SEVERAL DIFFERENT ASPECT RATIOS}

For meaningful comparisons of the behavior for several different aspect ratios we need to examine the behavior at their respective $\phi_{J}(\alpha)$. We therefore first consider the determination of $\phi_{J}(\alpha)$ before turning to analyses of the dependence on the aspect ratio.

\section{A. Approximate determinations of $\phi_{J}(\alpha)$}

The determination of $\phi_{J}(\alpha)$ is a difficult task and this is especially so for small asphericities. Our determination of the jamming density relies on the fact that the jamming density is the density where pressure and shear stress at small $\dot{\gamma}$ decay algebraically with $\dot{\gamma}$. This simple recipe is however difficult to use due to both corrections to scaling, finite size effects, and-especially for the case of small asphericities-a crossover from spherical behavior at larger shear rates to the true ellipsoidal behavior, as illustrated in Sec. III A above. Reliable determinations of $\phi_{J}$ need to take all these effects into account. We here chose to neglect all these complications and instead determine approximate values of $\phi_{J}(\alpha)$ as the densities where $p(\dot{\gamma} ; \alpha)$ behave similarly to $p(\dot{\gamma} ; 1.00)$. Behind this is the assumption that the exponent $q$ in $p \sim \dot{\gamma}^{q}$ for ellipsoids is not altogether different from the value for spheres; it does not entail the assumption that the models are in the same universality class.
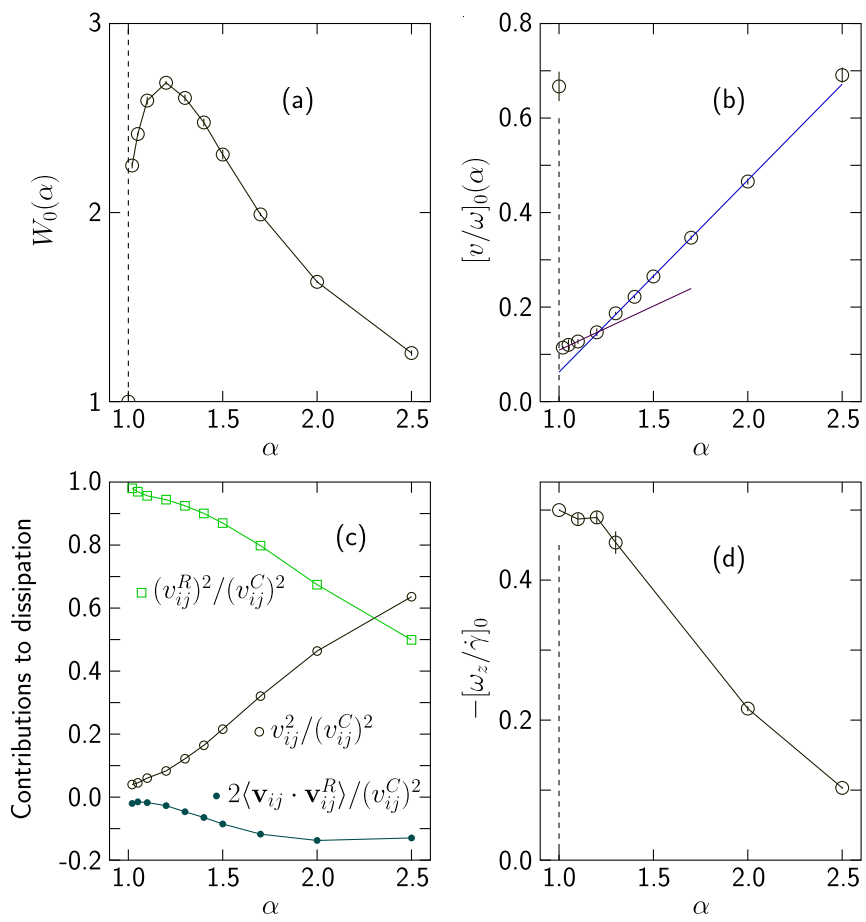

FIG. 6. Dependency on the aspect ratio, $\alpha$. All quantities are obtained after extrapolating to the $\dot{\gamma} \rightarrow 0$ limit at the respective $\phi_{J}(\alpha)$. Panels (a) and (b) are $W_{0}$ and $[v / \omega]_{0}$ from Fig. 3. Panel (c) relates to Fig. 4 and shows the relative contribution to the dissipation of the three terms in Eq. (5) vs $\alpha$. (The points for $\alpha=1.00$ are not included since they are far off and would only clutter the figure.) The data in panel (d) are particle rotations $\left[\omega_{z} / \dot{\gamma}\right]_{0}$ due to the shearing. These figures suggest a crossover to different behaviors at $\alpha \approx 1.2$.

Figure 5(a) shows $p$ vs $\dot{\gamma}$ both for spheres-big open circles-and for ten different $\alpha>1$ at the determined jamming densities $\phi_{J}(\alpha)$. We note that each $p(\dot{\gamma}, \alpha)$ is algebraic and to a decent approximation behaves the same as $p(\dot{\gamma}, 1)$. Panel (b) is $\phi_{J}(\alpha)$.

\section{B. Dependence on aspect ratio}

We now examine four different quantities at the different $\phi_{J}(\alpha)$ and extrapolated to the $\dot{\gamma} \rightarrow 0$ limit, as shown in Appendix D. Figure 6(a) shows that $W_{0}(\alpha)$-the relative rotation around the minor axes-first increases with increasing $\alpha$, reaches a maximum at $\alpha \approx 1.2$, then starts decreasing and is close to unity at $\alpha=2.5$. It is interesting to note that this peak in $W_{0}(\alpha)$ does not coincide with the maximum jamming density, which is at $\alpha \approx 1.5$ both in determinations from isotropically jammed packings $[28,29]$ and in the present study. In panel (b) $[v / \omega]_{0}$ shows a change from one linear region to another at $\alpha \approx 1$. In panel (c) which shows the relative contribution of the different terms in Eq. (5)—excluding $\alpha=1$, which is altogether different-a similar change is also present, though not equally sharp. Panel (d) shows that the rotational velocity around the $z$ axis (of the laboratory frame) associated with the shearing, which is $-\left[\omega_{z} / \dot{\gamma}\right]_{0}=1 / 2$ for spheres, first stays almost constant but then starts decreasing for $\alpha>1.2$. The very low rotational velocity at $\alpha=2.5$ is presumably due to the difficulty for very elongated particles to 
find room to rotate in dense configurations. A decrease of $\omega_{z}$ with increasing asphericity has also been found in simulations of spherocylinders [20].

The conclusion of a change in behavior at $\alpha \approx 1.2$ may actually also be drawn from Fig. 5(a), which is $p$ vs $\dot{\gamma}$ for several $\alpha$ at their respective $\phi_{J}(\alpha)$. By focusing on $p$ at any constant $\dot{\gamma}$ it then appears that $p$ first increases, then reaches a maximum at $\alpha=1.2$ or 1.3 , and eventually decreases as $\alpha$ increases further. This is thus in agreement with the conclusions of Fig. 6. It should however be mentioned that this analysis of $p$ is not quite as conclusive since pressure is a quantity that does depend strongly on $\phi$, which means that our uncertainty in the $\phi_{J}(\alpha)$ could be important. This means that we cannot rule out the possibility of a differently looking curve if the data had instead been taken at the true $\phi_{J}(\alpha)$ rather than at the $\phi_{J}(\alpha)$ from our approximate determination.

\section{SUMMARY AND DISCUSSION}

To summarize, we have shown that a change from spherical to slightly ellipsoidal particles with aspect ratio $\alpha=1.02$ gives an altogether different microscopic dynamics. Comparing spheres and ellipsoids close to jamming it is found that the translational velocity is reduced by $80 \%$, and that the rotations get a different role for the ellipsoids, contributing to the particles ability to fit together. The dramatic difference is also seen when separating the dissipation into contributions from the translational and the rotational velocities. For spheres the dissipation is dominated by the translational motion, whereas the dissipation in the ellipsoids is instead dominated by the rotations.

What can now be concluded about the relation between this different dynamics and the quartic modes? To settle that question beyond possible doubt would require a study where both the quartic modes and the microscopic dynamics were determined simulataneously, which is well beyond the scope of the present work. When trying to find an explanation for the present findings from properties of isotopically jammed systems, it does however seem that the presence of quartic modes is a natural candidate simply because they are the outstanding difference between packings of ellipsoids and packings of spheres. What gives additional credibility to such a conclusion is the observation that this different dynamics very strongly affects the rotations, which is in agreement with the quartic modes being primarily rotational in character [21,22]. We therefore conclude that there is strong evidence for considering the very different dynamics in sheardriven ellipsoids to be effects of the quartic vibrational modes on the shear-driven systems.

A second finding is the change in behavior of several quantities at $\alpha \approx 1.2$, which suggests a crossover at this aspect ratio. We believe that this is an effect of a competition between two different mechanisms, where the first would give an increase of the rotational motion with increasing $\alpha$ as a means of fitting into the constantly changing environment and the second is related to the difficulty of very elongated particles to find room to rotate in dense packings, which would give a decrease in the rotational velocity for larger $\alpha$.

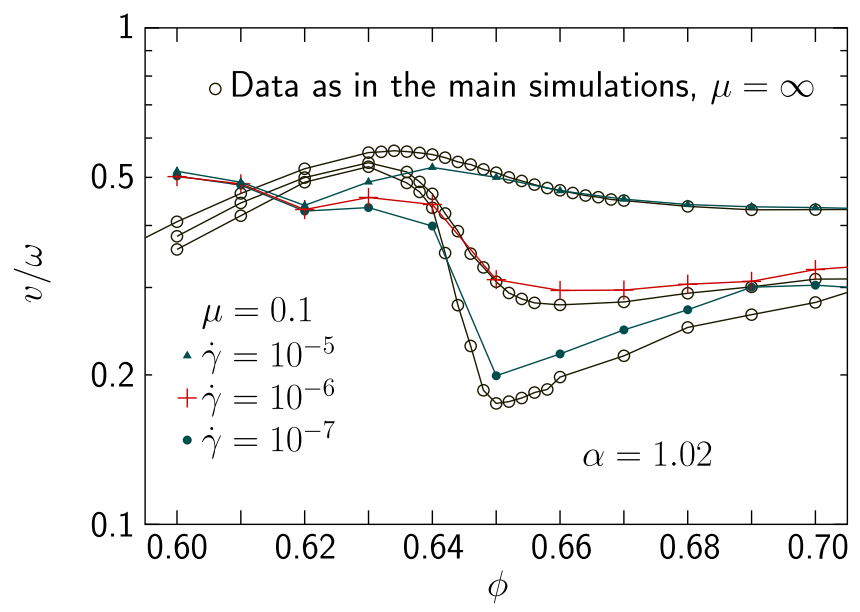

FIG. 7. Comparison of different kinds of dissipative forces. In dry friction the tangential dissipative force is limited through $F_{t}^{\text {dis }} \leqslant$ $\mu F^{\mathrm{el}}$. We here compare our data from the main simulations-which may be described as being produced with $\mu=\infty$-with simulations with $\mu=0.1$. At and close to the transition at $\phi \approx 0.652$ there are very small changes, though some differences start to be visible below $\phi=0.62$.

\section{ACKNOWLEDGMENTS}

We thank S. Teitel for many discussions. The simulations were performed on resources provided by the Swedish National Infrastructure for Computing (SNIC) at HPC2N.

\section{APPENDIX A: TANGENTIAL DISSIPATION AS IN DRY FRICTION}

Our model is a generalization of the model in Ref. [31] to noncircular particles. This model is one of the simplest possible that includes both contact dissipation and rotation. An undesired feature of this model is however that the tangential part of the dissipation at a given contact may jump discontinuously to zero when the particles lose contact. With a parameter $\mu$ that controls the maximum size of the dissipation, $F_{t}^{\text {dis }} \leqslant \mu F^{\mathrm{el}}$, as in dry friction, our model may be described as having $\mu=\infty$ since any contact, however weak, will be able to sustain an arbitrarily big dissipative force, given by the velocity difference at the point of contact.

Since the model with $\mu=\infty$ can be argued to be unphysical we have performed additional simulations with finite $\mu$. It was then found necessary to take a value as low as $\mu=0.1$ to get any clearly visible changes. Figure 7 is therefore a comparison of $v / \omega$ obtained with $\mu=0.1$ and the data in the paper, which are for $\mu=\infty$. Even with such a small $\mu$ there are no significant differences at the density of interest in our studies, which is around $\phi=0.65$. The only notable difference is a dip in $v / \omega$ below $\phi=0.62$, which is well outside of our region of interest. Looking into the reason for this weak dependence on $\mu$, one finds that the tangential dissipative forces are typically much smaller than the elastic forces, at the densities of interest, which means that taking $\mu=0.1$ only affects a small fraction of contacts with the weakest elastic forces. We therefore conclude that the results and the analyses of the present paper would not be significantly affected by 

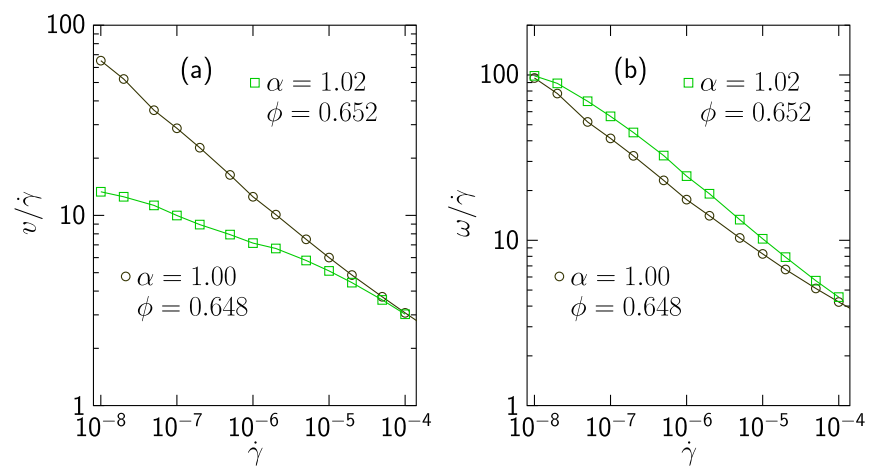

FIG. 8. Translational and rotational velocities for ellipsoidal and spherical particles. The data are shown at their respective $\phi_{J}$, $\phi_{J}(1.00)=0.648$ and $\phi_{J}(1.02)=0.652$. Panel (a) shows that the nonaffine translational velocity at low shear strain rates is much lower for ellipsoids than for spheres. The rotational velocity, panel (b), is in contrast somewhat higher for ellipsoids than for spheres. These behaviors of $v$ and $\omega$ together give a dramatic decrease in $v / \omega$, shown in Figs. 3(b) and 3(c).

instead using a model that couples the elastic and the dissipative forces.

\section{APPENDIX B: TRANSLATIONAL AND ROTATIONAL VELOCITIES}

To look into the origin of the dip in $v / \omega$ in Fig. 3(b) we now examine $v$ and $\omega$ for spheres and ellipsoids with $\alpha=1.02$ at the densities $\phi_{J}(1.00)=0.648$ and $\phi_{J}(1.02)=$ 0.652. Figures 8(a) and 8(b) show $v / \dot{\gamma}$ and $\omega / \dot{\gamma}$ and we first note that these quantities - open circles in panels (a) and (b) - behave the same for $\alpha=1.00$, which is consistent with $v / \omega$ being essentially independent of $\dot{\gamma}$. In comparison, for $\alpha=1.02-$ open squares in panels (a) and (b) $-v / \dot{\gamma}$ increases more slowly than $\omega / \dot{\gamma}$, which explains the decrease of $v / \omega$. The figure shows that it is the lower translational velocity which is the main reason for the small $v / \omega$ of the ellipsoids.

The velocities in Fig. 8 are single-particle quantities. To complement this, Fig. 9(a) shows the root-mean-square velocity difference at contact, $v_{i j}^{C}$, which can be argued to be a physically more relevant quantity than the single-particle velocities due to the relation to shear viscosity from power balance, $N k_{d}(z / 2)\left(v_{i j}^{C}\right)^{2}=\sigma \dot{\gamma} V$ [32]. Panel (a) shows these quantities at their respective $\phi_{J}(\alpha) . v_{i j}^{C}$, and thus also the shear viscosity, is found to be slightly bigger for ellipsoids than for spheres. (The contact number $z$, which enters the relation to the shear viscosity, is also different for the two cases, but these differences are too small to significantly affect the comparison.) Figure 9(b) is the same quantity at the same density, $\phi=0.648$, for comparison, which shows that the shear viscosity at the same density and small $\dot{\gamma}$ is indeed lower for the ellipsoids, consistent with expectations since $\phi_{J}(1.02)>\phi_{J}(1.00)$.

\section{APPENDIX C: ANTICORRELATION OF $v_{i j}$ AND $v_{i j}^{R}$}

Figure 4 shows an analysis of the dissipation at contacting particles which was done by splitting up the velocity
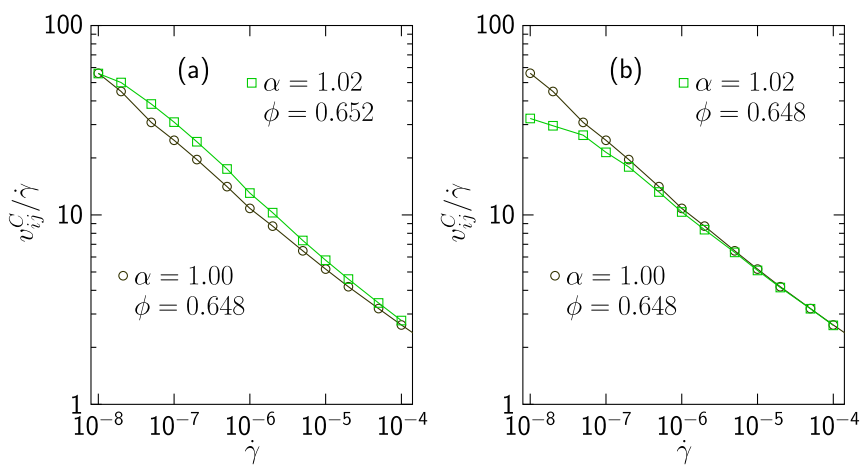

FIG. 9. Velocity difference at the point of contact. This is a quantity which is related to the dissipation and thereby to the shear viscosity. Panel (a) shows the behavior of spheres and ellipsoids at their respective $\phi_{J}$. Panel (b) which is data at the same $\phi$ for both cases is included to show that the ellipsoids, at a given density, have lower contact velocities than the spheres, and thereby also a lower shear viscosity.

difference on translational and rotational velocity differences. We here make use of the same data to extract information in a different way.

The quantity in focus is the correlation coefficient, which is obtained from the second term in Eq. (5), but with a different normalization,

$$
g^{\mathrm{RT}}=\frac{\left\langle\mathbf{v}_{i j} \cdot \mathbf{v}_{i j}^{R}\right\rangle}{v_{i j} v_{i j}^{R}} .
$$

Here $v_{i j}$ and $v_{i j}^{R}$ are the root-mean-square values.

Figure 10 shows that both spheres and ellipsoids have a region with almost perfect anticorrelation, $g^{\mathrm{RT}} \approx-0.996$, for

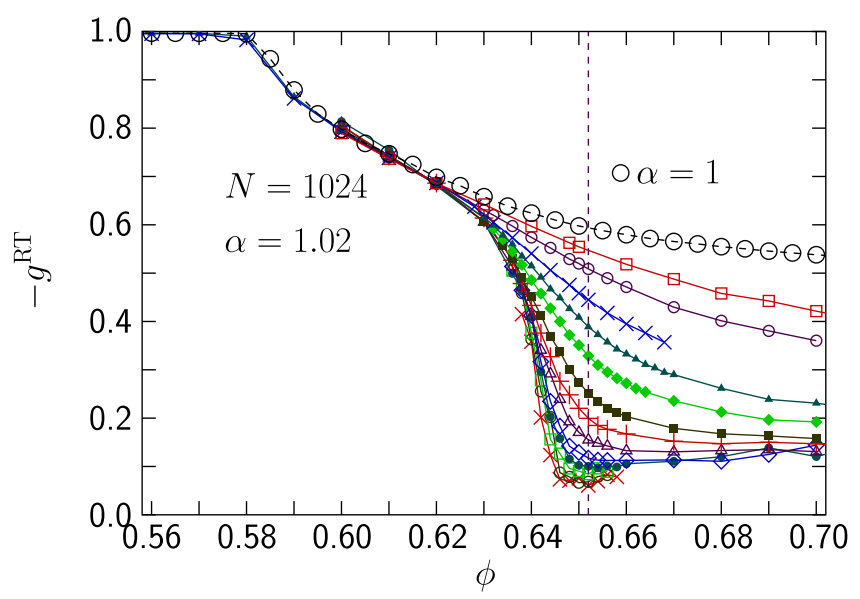

FIG. 10. Correlations between different contributions to the contact velocity difference for both spheres (one set of open circles) and ellipsoids for several different shear rates. The figure shows the (anti)correlations between the translational contribution $\mathbf{v}_{i j}$ and the rotational contribution $\mathbf{v}_{i j}^{R}$ to the velocity difference at contacts. It is found that $\mathbf{v}_{i j}$ and $\mathbf{v}_{i j}^{R}$ are almost perfectly anticorrelated at low densities, $\phi<0.58$, which means that the rotations almost entirely compensate for the translational velocity differences. For the spherical particles $\mathbf{v}_{i j}$ and $\mathbf{v}_{i j}^{R}$ remain strongly anticorrelated but, for the aspherical particles at the lowest shear rates, these correlations almost vanish and the two contributions are almost independent. 

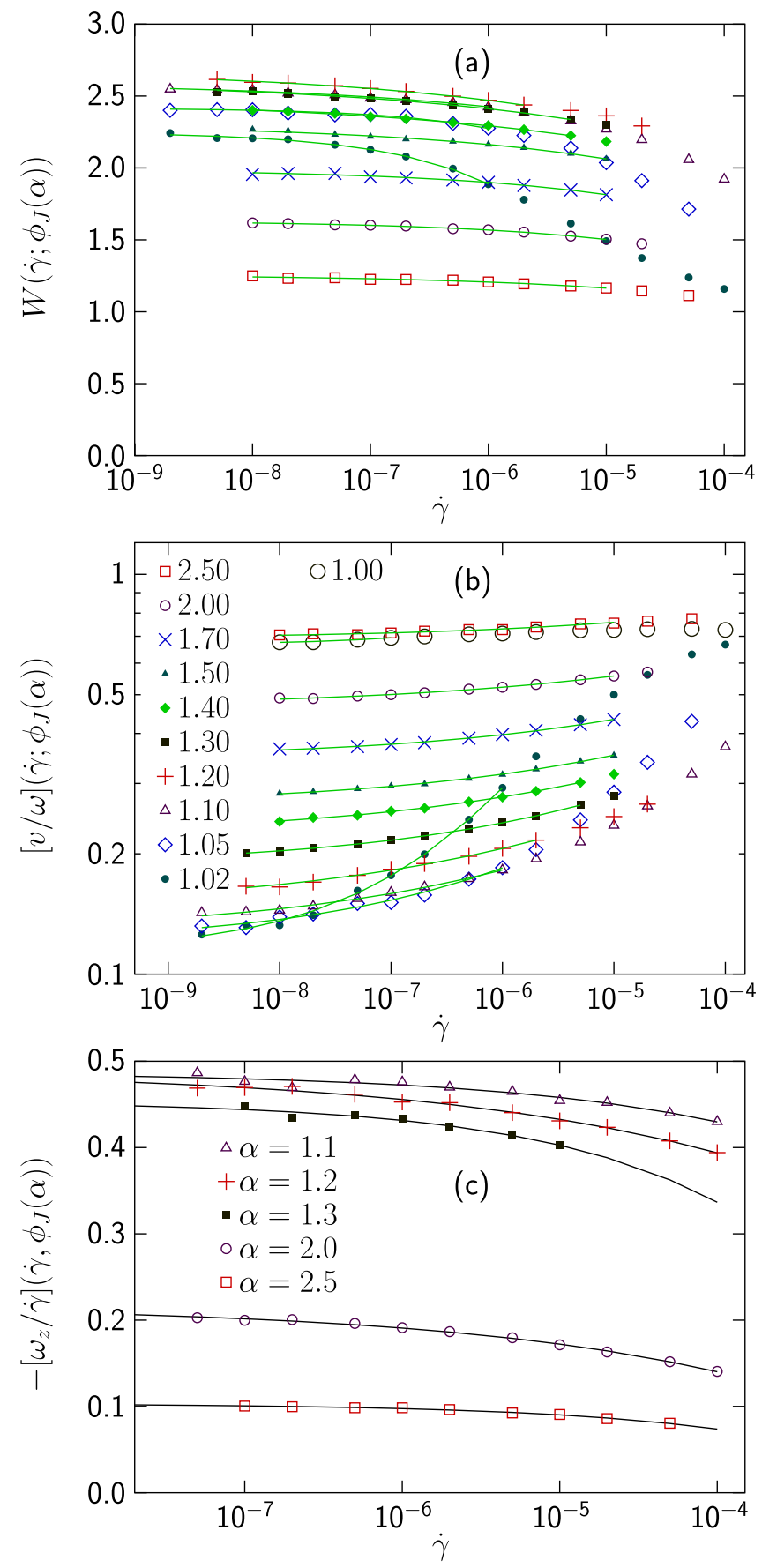

FIG. 11. Extrapolations behind the data in Figs. 6(a), 6(b), and $6(\mathrm{~d})$. $\phi<0.58$. (Note the different density range; $\phi$ in Fig. 4 only extends down to $\phi=0.60$.) In this region there is Bagnold scaling, $p \sim \dot{\gamma}^{2}$, and the particles have on average less than two contacts, which means that rotations can almost altogether compensate for the translational velocity differences. There is then a jump to a region with Newtonian behavior, $p \sim \dot{\gamma}$, and a larger number of contacts, $z>4$. It is then no longer possible for the particles to eliminate the velocity differences by rotations, and the anticorrelation is weaker, down to $-g^{\mathrm{RT}} \approx 0.6$ around the jamming density. Just as in the other quantities discussed above, there is essentially no $\dot{\gamma}$ dependence for the spherical particles, and the correlation coefficient is therefore shown for $\dot{\gamma}=10^{-5}$, only.

In Ref. [31], Fig. 21, this correlation was explored in a 2D model by means of a scatter plot which suggested an essentially perfect anticorrelation at low densities [panel (a)] changing to a weaker anticorrelation at higher densities [panel (b)]. (In the notation of Ref. [31] $V_{i j, T}$ is the tangential component of the translational velocity difference, which is almost the same as the full translational velocity difference, here denoted by $\mathbf{v}_{i j}$.)

As jamming is approached the behavior of ellipsoids again differs considerably from the behavior of spheres; the correlation coefficient drops dramatically. An extrapolation to the $\dot{\gamma} \rightarrow 0$ limit by fitting $g^{\mathrm{RT}}$ at $\phi_{J}$ to an algebraic behavior plus a constant gives $-g_{0}^{\mathrm{RT}}=0.045$, which implies that $\mathbf{v}_{i j}$ and $\mathbf{v}_{i j}^{R}$ are almost entirely decoupled from each other, in sharp contrast to the essentially perfect anticorrelation at low densities.

\section{APPENDIX D: EXTRAPOLATIONS TO THE $\dot{\gamma} \rightarrow 0$ LIMIT}

Figure 11 shows extrapolations of three different quantities by fitting to constant plus algebraic behavior. The corresponding constants, $W_{0},[v / \omega]_{0}$, and $[\omega / \dot{\gamma}]_{0}$, are shown in Figs. 6(a), 6(b), and 6(d).

A remark is in order regarding the determination of $\omega_{z} / \dot{\gamma}$ shown in Fig. 11(c): the constant shearing of the system in the $x-y$ plane leads to a rotational velocity around the $z$ axis which for spheres is given by $\omega_{z}=-\dot{\gamma} / 2$. This rotation does however turn out to be a small signal compared to the erratic particle rotations, and this makes precise determinations of $\omega_{z}$ difficult. To handle this we have here taken data from some additional runs with $N=65536$ particles that give better statistics. These runs have however only been done for a few different $\alpha$, and this is the reason why there are only five curves in Fig. 11(c).
[1] A. Donev, S. Torquato, and F. H. Stillinger, Phys. Rev. E 71, 011105 (2005).

[2] L. Berthier and T. A. Witten, Phys. Rev. E 80, 021502 (2009).

[3] D. Vågberg, P. Olsson, and S. Teitel, Phys. Rev. E 83, 031307 (2011).

[4] M. Ozawa, L. Berthier, and D. Coslovich, SciPost Phys. 3, 027 (2017).

[5] C. S. O’Hern, S. A. Langer, A. J. Liu, and S. R. Nagel, Phys. Rev. Lett. 88, 075507 (2002).
[6] C. S. O’Hern, L. E. Silbert, A. J. Liu, and S. R. Nagel, Phys. Rev. E 68, 011306 (2003).

[7] D. Vågberg, D. Valdez-Balderas, M. A. Moore, P. Olsson, and S. Teitel, Phys. Rev. E 83, 030303(R) (2011).

[8] C. Heussinger and J.-L. Barrat, Phys. Rev. Lett. 102, 218303 (2009).

[9] C. Heussinger, P. Chaudhuri, and J.-L. Barrat, Soft Matter 6, 3050 (2010).

[10] P. Olsson and S. Teitel, Phys. Rev. Lett. 99, 178001 (2007). 
[11] T. Hatano, J. Phys. Soc. Jpn. 77, 123002 (2008).

[12] T. Hatano, J. Phys.: Conf. Ser. 319, 012011 (2011).

[13] M. Otsuki and H. Hayakawa, Phys. Rev. E 80, 011308 (2009).

[14] T. Hatano, Phys. Rev. E 79, 050301(R) (2009).

[15] T. Hatano, Prog. Theor. Phys. Suppl. 184, 143 (2010).

[16] B. P. Tighe, E. Woldhuis, J. J. C. Remmers, W. van Saarloos, and M. van Hecke, Phys. Rev. Lett. 105, 088303 (2010).

[17] T. Marschall, Y.-E. Keta, P. Olsson, and S. Teitel, Phys. Rev. Lett. 122, 188002 (2019).

[18] T. Marschall and S. Teitel, Phys. Rev. E 97, 012905 (2018).

[19] T. A. Marschall and S. Teitel, Phys. Rev. E 100, 032906 (2019).

[20] T. A. Marschall, D. Van Hoesen, and S. Teitel, Phys. Rev. E 101, 032901 (2020).

[21] M. Mailman, C. F. Schreck, C. S. O'Hern, and B. Chakraborty, Phys. Rev. Lett. 102, 255501 (2009).

[22] C. F. Schreck, M. Mailman, B. Chakraborty, and C. S. O'Hern, Phys. Rev. E 85, 061305 (2012).
[23] T. Börzsönyi, B. Szabó, S. Wegner, K. Harth, J. Török, E. Somfai, T. Bien, and R. Stannarius, Phys. Rev. E 86, 051304 (2012).

[24] D. B. Nagy, P. Claudin, T. Börzsönyi, and E. Somfai, Phys. Rev. E 96, 062903 (2017).

[25] M. Trulsson, J. Fluid Mech. 849, 718 (2018).

[26] J. C. Maxwell, Philos. Mag. 27, 294 (1864).

[27] A. Zaccone and E. Scossa-Romano, Phys. Rev. B 83, 184205 (2011).

[28] A. Donev, I. Cisse, D. Sachs, E. A. Variano, F. H. Stillinger, R. Connelly, S. Torquato, and P. M. Chaikin, Science 303, 990 (2004).

[29] A. Donev, R. Connelly, F. H. Stillinger, and S. Torquato, Phys. Rev. E 75, 051304 (2007).

[30] P. Olsson, Phys. Rev. Lett. 122, 108003 (2019).

[31] D. Vågberg, P. Olsson, and S. Teitel, Phys. Rev. E 95, 052903 (2017).

[32] I. K. Ono, S. Tewari, S. A. Langer, and A. J. Liu, Phys. Rev. E 67, 061503 (2003). 